\title{
Raman and Infrared Characterization of Gadolinium-doped Manganese Sulfide
}

\author{
Gerasimova Yu.V..$^{1,2}$, Oreshonkov A.S. ${ }^{1,2}$, Romanova O.B. ${ }^{1,3}$, \\ Ivanenko A.A. ${ }^{1}$, Krylov A.S. ${ }^{1}$
}

${ }^{1}$ Kirensky Institute of Physics (Krasnoyarsk, Russia)

${ }^{2}$ Siberian Federal University (Krasnoyarsk, Russia)

${ }^{3}$ Siberian State Aerospace University M F Reshetnev (Krasnoyarsk, Russia)

oreshonkov@iph.krasn.ru

\begin{abstract}
Gadolinium manganese sulfide solid solutions are investigated by infrared and Raman spectroscopy. Longitudinal optical-transverse optical splitting of the manganese-sulfur bond vibration is observed in vibrational spectra. The Raman spectra contain modes that are prohibited in crystal structure of alpha phase of manganese sulfide, which are associated with activation of the phonons from both Brillouin zone center and its $\mathrm{X}$ and $\mathrm{L}$ points. The concentration dependence of transverse optical and longitudinal optical modes' frequencies is calculated within the frame of modified random-element-isodisplacement model, being in good
\end{abstract}


agreement with experiment. Both theory and experiment admit that solid solution under study exhibits "one-mode" behavior.

\section{Introduction}

Nowadays, great attention of the researchers is paid to the investigation of materials with strong interconnection between electrical and magnetic properties, in the relation to future applications in devices of next generation electronics (1). According to the earlier results $(2,3)$, from the point of view of fundamental research, the special attention is to be devoted to transition metal sulfide compounds containing rare earth elements, e. g. $R e_{\mathrm{x}} \mathrm{Mn}_{1-\mathrm{x}} \mathrm{S}$ where $R e=\mathrm{Gd}, \mathrm{Sm}$, Ho, exhibiting phase transitions between metal and insulator, accompanied by the conductivity type change from p-type to n-type, by the magnetic phase transformations including variations of magnetic properties within the same magnetic symmetry, providing the possibility of magnetoresistance effect under certain conditions. However, despite certain advances in the study of these compounds (4-7), the vibrational spectra of $R e$-doped manganese sulfides are studied still insufficiently.

Doping of manganese sulfide with gadolinium leads to formation of solid solution preserving face-centered crystal lattice of sodium chloride type. Magnetic ion $\mathrm{Mn}^{2+}$ is in the crystal field of octahedral symmetry created by sulfur 
environment. With increasing degree of cation substitution $x$ the parameter of the unit cell grows (2). Doping with rare earth gadolinium ion leads to local deformation of the structure associated with the difference in the ionic radii of the manganese and gadolinium ( $r=0.83 \AA$ for manganese, $r=0.94 \AA$ for gadolinium).

Raman and infrared spectroscopy are important tools for the study of the relationship of the physical properties and structure of the matter. Therefore, it is attractive to study the influence of compositional change onto the phonon spectra for gadolinium-doped manganese sulfide. Similar solid solutions are of interest from the point of view of manifestation of so-called "one-mode" or "two-mode" behavior type (8). In the class of solid solutions with “one-mode” behavior, each of the optical modes' frequencies with $k=0$ (infrared or Raman-active) varies continuously and approximately linearly with solid solution content between the frequencies characteristic to the end members of solid solution system (9). In the class of solid solutions with "two-mode" behavior, Raman lines intensity of these modes is proportional to the content every constituent. Varying the content leads to intensity growth of the one mode and to decrease of another mode, while the modes’ frequencies remain practically unchanged. 
In this paper we discuss vibrational spectra of $\mathrm{Gd}_{x} \mathrm{Mn}_{1-x} \mathrm{~S}(x=0.04,0.1$, 0.25) solid solutions; obtained values were compared to those calculated using the modified random-element-isodisplacement (9) model.

\section{Experimental}

The $\mathrm{Gd}_{x} \mathrm{Mn}_{1-x} \mathrm{~S}$ crystals were synthesized in a quartz reactor from a melt of polycrystalline powders of sulfides. The reactor with a charge in glass-carbon crucibles was pulled through a single-turn inductor of a high-frequency facility. As an inert medium, high-purity argon was used (10). $\mathrm{Gd}_{x} \mathrm{Mn}_{1-x} \mathrm{~S}$ solid solution remains a single phase system with the studied gadolinium content range. $x=0.25$ is a critical concentration exceeding of which results in appearance of extra phases verified by additional peaks in X-ray diffraction patterns. These additional peaks are indexed as those of GdS system.

Analysis of scanning electron microscope images obtained with Hitachi TM3000 microscope proves that the sample is featured by a microinhomogeneity associated with slight variation of gadolinium content. All the investigated samples are polycrystals. 
Raman spectra were recorded using T64000 spectrometer (Horiba Jobin Yvon, France) in backscattering geometry, with spectral resolution $2 \mathrm{~cm}^{-1}$ in the range of $30-750 \mathrm{~cm}^{-1}$. Argon laser at wavelength $\lambda=514.5 \mathrm{~nm}$ was used as a source of excitation radiation. The radiation power at the sample was $<5 \mathrm{~mW}$. Fourier spectrometer VERTEX 80V (BRUKER, Germany) was used to obtain the far IR (infrared) absorption spectra. IR spectra were obtained in the spectral range of $30-700 \mathrm{~cm}^{-1}$ at $0.2 \mathrm{~cm}^{-1}$ spectral resolution. Temperature scanning was performed using Optistat TM AC-V 12a cryostat (Oxford instruments, UK) within range from 5 to $330 \mathrm{~K}$.

To perform the lattice dynamics simulation of pure $\alpha-\mathrm{MnS}$ and gadoliniumdoped manganese sulfides the program package LADY (Smirnov, M. B.; Kazimirov, V.Yu. Dubna, Russia) was used. The atomic vibration frequencies were obtained using the modified random-element-isodisplacement model (9). Then we simulated complete spectra of the crystals; interionic interactions between Mn-S, Gd-S and S-S have been described in the fairly common model of "rigidion”, where interatomic potential is considered as a sum of long range Coulomb electrostatic:

$V\left(r_{i j}\right)=\frac{1}{2} \sum_{i, j} \frac{Z_{i} Z_{j}}{r_{i j}}+U\left(r_{i j}\right)$, 
the short-range interaction potential was taken in the Buckingham form:

$$
U\left(r_{i j}\right)=\lambda \exp \left(-r_{i j} / \rho\right)-C / r_{i j}{ }^{6},
$$

where $r_{\mathrm{ij}}$ is the interatomic distance and $\lambda, \rho$ and $C$ are the parameters characterizing of the short-range pair interionic interaction. To find model parameters, the special optimization program was written (Oreshonkov A. S.,

Krasnoyarsk, Russia) and tested for several compounds from different chemical classes (11-26).

\section{Results and discussion}

According to the group theory analysis for $\alpha-\mathrm{MnS}$ (point symmetry group $\mathrm{O}_{\mathrm{h}}$ ), vibrational representation in the center of the Brillouin zone is as follows:

$$
\Gamma_{\text {vibr }}=2 T_{1 u}
$$

and one of the triply degenerate modes is acoustic, while the other one is optical. For gadolinium manganese sulfide solid solutions, additional $T_{1 u}$ mode should appear in the vibrational representation. This mode is so-called "gap” mode (9), which is related to Gd - S bond vibration. According to the selection rules, all the modes noted above are IR active, but not active in Raman. Despite these considerations, we obtained Raman spectra form these solid solutions. 
Figure 1 shows IR and Raman spectra of $\mathrm{Gd}_{x} \mathrm{Mn}_{1-x} \mathrm{~S}(x=0.04,0.1,0.25)$ which are compared with pure $\alpha-\mathrm{MnS}$. IR spectra practically coincide in position and shape with the data for natural mineral Alabandite $(\alpha-M n S)(27,28)$, Raman spectrum of $\alpha$-MnS coincides with the spectrum form RRUFF database (29). The spectra in Fig.1 are obtained in the equivalent experimental conditions and later they were normalized by the spectral contour area.

Observed frequencies of vibrational modes in Raman and infrared spectra correspond to the phonons emerging from the center of Brillouin zone, as presented in Table 1.

The rest of peaks observable in the Raman spectra in Fig. 1 are the vibrational modes emerging from the boundaries of Brillouin zone. Phonon dispersion curves along the $\mathrm{W}-\mathrm{L}-\Gamma-\mathrm{X}-\mathrm{W}-\mathrm{K}-\Gamma$ points of the Brillouin zone and phonon density of states for $\alpha-\mathrm{MnS}$ are presented in (30).

Emerging of vibrational modes from the Brillouin zone boundaries can be produced either by the defects of crystal structure or by the local distortions of that structure induced by the Gd ions' doping. Spectral contour at $274 \mathrm{~cm}^{-1}$ may be associated with activation of the LO (L) and LO (X) phonons. The peaks in the region of $200 \mathrm{~cm}^{-1}$ correspond to TO (L) and LA (L) vibrations. In the frequency 
range below $180 \mathrm{~cm}^{-1}$ manifestation of LA (X), TA (L) and TA (X) modes is possible (30).

In addition to the features in Raman spectra discussed above, a wide bellshaped band is observed near $600 \mathrm{~cm}^{-1}$ in Raman spectra of $\alpha-\mathrm{MnS}$ and (Mn,Gd)S solid solutions. The intensity of this band is maximum for $\alpha$-MnS and drops down with gadolinium concentration. We suppose to attribute it to double resonance of phonons from Brillouin zone boundaries, in analogy with graphite Raman spectra (31). Increase of Gd content must lead to the decrease of anharmonicity of $\mathrm{Mn}-\mathrm{S}$ vibrations and to observed decrease of this band height.

The calculated and measured TO and LO optical phonon modes are summarized in Fig. 2. On the whole, the predicted phonon frequencies are in good agreement with the experimental data.

The interpretation of experimental data within the MREI model evidences that for the solid solution under study the general situation of "one-mode" behavior is implemented. TO and LO optical mode frequencies vary continuously and approximately linearly with Gd concentration from the frequency of $\mathrm{MnS}$ to that of GdS (32), which are also shown in Fig. 1 by dashed vertical lines. 
Calculations show presence of the triply degenerate gap mode of $\mathrm{Gd}$ vibration which is split into two modes - the transverse and the longitude ones. Increase of Gd content results in the increase of gap mode splitting. The crystal structure of $\mathrm{Gd}_{x} \mathrm{Mn}_{1-x} \mathrm{~S}$ preserves stability up to $x=0.25$. Upon further increase of Gd content, the system becomes less stable, and it is more energetically favorable for this system to release the excess of gadolinium in the form of GdS. TO and LO gap modes of solid solution at $x$ above 0.25 are transformed into TO and LO modes of pure GdS, as shown in Fig. 2 by the bending of dashed lines.

At excessive power of the laser beam the formation of burnt craters on the surface of (Gd,Mn)S samples is observed. In analogy with paper (33), we observe disappearance of Raman peaks corresponding to $\mathrm{Gd}_{0.1} \mathrm{Mn}_{0.9} \mathrm{~S}$ structural vibration are practically absent at burnt spots, and Raman spectra recoded from these spots correspond to known spectra of $\mathrm{MnO}_{2}$ (34), Fig. 3 .

No anomalies due to restructuring were revealed upon decrease of the temperature, and all solid solutions preserve $\mathrm{O}_{\mathrm{h}}$ symmetry down to $5 \mathrm{~K}$.

\section{Conclusion}

We investigated solid solutions of $\mathrm{Gd}_{x} \mathrm{Mn}_{1-x} \mathrm{~S}$ ( $\left.x=0.25,0.1,0.04\right)$ using infrared absorption and Raman scattering. LO-TO splitting of the manganese- 
sulfur bond vibration is observed in vibrational spectra. The Raman lines forbidden by the selection rules are manifested in the spectra due to the violation of the local symmetry of the crystal. The solid solutions under study exhibit "one-mode" behavior that was successfully described by MREI model. Critical concentration of gadolinium for preserving the stability of the cubic MnS crystal lattice is 0.25.

\section{Acknowledgments}

The authors are thankful to Dr. A.V. Shabanov for scanning electron microscopy study of the samples. The reported study was funded by Russian Foundation for Basic Research according to the research project No. 16-32-00351 mol_a. 


\section{References}

1. Ehrenstein, W.; Mazur, N. D.; Scott. J. F. Multiferroic and magnetoelectric materials. Nature 2006, 442, 759-765.

2. Romanova, O. B.; Ryabinkina, L. I.; Sokolov, V. V.; Pichugin, A. Yu.; Velikanov, D. A.; Balaev, D. A.; Galyas, A. I.; Demidenko, O. F.; Makovetskii, G. I.; Yanushkevich, K. I. Magnetic properties and the metal-insulator transition in $\mathrm{Gd}_{\mathrm{X}} \mathrm{Mn}_{1-\mathrm{X}} \mathrm{S}$ solid solutions. Solid State Communications 2010, 150, 602-604.

3. Aplesnin, S.; Romanova, O.; Harkov, A.; Balaev, D.; Gorev, M.; Vorotinov, A.; Sokolov, V.; Pichugin, A. Metal-semiconductor transition in $\mathrm{Sm}_{\mathrm{X}} \mathrm{Mn}_{1-\mathrm{X}} \mathrm{S}$ solid solutions. Physica status solidi (b) 2012, 249, 812-817.

4. Yanushkevich, K. I. Solid solutions monochalcogenides 3d-elements; Varaksin: Minsk, Belarus, 2009; 256 pp.

5. Burlet, P. Etude par diffraction neutronique des ètats d’ordere magn ètique dans les solutions solides nuclèairement dèsordonnèes du type $M n_{X} M_{1-X} S$ $(\mathrm{M}=\mathrm{V}, \mathrm{Cr}, \mathrm{Fe}, \mathrm{Co}, \mathrm{Ni})$ Le titre de docteur ès-sciences physiques; De L Universitè de Grenoble, 1968; 94 pp.

6. Vaughan, D.; Craig, J. Chemistry of Sulfide Materials; Mir Press: Moscow, Russia, 1981, 575 pp. 
7. Golubkov, A. V.; Goncharova, E. V.; Zhuze, V. P.; Loginov, G. M.; Sergeeva, V. M. Physical Properties of Chalcogenides of Rare-Earth Elements; Nauka Publishers: Leningrad, Russia, 1973, 303 pp.

8. Elliott R. J.; Ipatov I. P. Optical Properties of Mixed Crystals; NorthHolland Publ. Co.: Amsterdam, Holland, 1988, 438 pp.

9. Chang, I. F.; Mitra, S. S. Application of a Modified Random-ElementIsodisplacement Model to Long-Wavelength Optic Phonons of Mixed Crystals. Physical Review 1968, 172, 924 - 933.

10. Aplesnin, S. S.; Ryabinkina, L. I.; Romanova, O. B.; Sokolov, V. V.; Pichugin, A. Yu.; Galyas, A. I.; Demidenko, O. F.; Makovetskiı̌, G. I.; Yanushkevich, K. I. Magnetic and electrical properties of cation-substituted sulfides $\mathrm{Me}_{\mathrm{x}} \mathrm{Mn}_{1-\mathrm{x}} \mathrm{S}(\mathrm{Me}=\mathrm{Co}, \mathrm{Gd})$. Physics of the Solid State 2009, 51, 698-701.

11. Lim, C. S.; Aleksandrovsky, A. S.; Molokeev, M. S.; Oreshonkov, A. S.; Ikonnikov, D. A.; Atuchin, V.V., Triple molybdate scheelite-type upconversion phosphor $\mathrm{NaCaLa}\left(\mathrm{MoO}_{4}\right)_{3}: \mathrm{Er}^{3+} / \mathrm{Yb}^{3+}$ : structural and spectroscopic properties. Dalton Transactions 2016, 45, 15541-15551.

12. Krylov, A. S.; Molokeev, M. S.; Misyul, S. V.; Krylova, S. N.; Oreshonkov, A. S.; Ivanenko, A. A.; Zykova, V. A.; Ivanov, Y. N.; Sukhovsky, A. 
A.; Voronov, V. N.; Safonov, I. N. and Vtyurin, A. N. Crystal structure and phase transitions of a layered perovskite-like $\mathrm{CsScF}_{4}$ crystal. CrystEngComm 2016, 18, 8472-8486.

13. Strikina, E. A.; Krylov, A. S.; Oreshonkov, A. S. and Vtyurin, A. N. Raman scattering study of $\delta-\mathrm{BiB}_{3} \mathrm{O}_{6}$ crystal. Ferroelectrics 2016, 501(1), 26-31.

14. Oreshonkov, A. S.; Krylov, A. S.; Shestakov, N. P.; Voronov, V. N.; Ershov, A. A.; Strikina, E. A. and Vtyurin, A. N. Vibrational spectra of $\mathrm{NdF}_{3}$ crystal. Ferroelectrics 2016, 501 (1), 15-19.

15. Lim, C. S.; Atuchin, V. V.; Aleksandrovsky, A. S.; Molokeev M. S.; Oreshonkov, A. S. Incommensurately modulated structure and spectroscopic properties of $\mathrm{CaGd}_{2}\left(\mathrm{MoO}_{4}\right)_{4}: \mathrm{Ho}^{3+} / \mathrm{Yb}^{3+}$ phosphors for up-conversion applications. Journal of Alloys and Compounds 2016, doi: 10.1016/j.jallcom.2016.06.134.

16. Oreshonkov, A. S.; Gerasimova, J. V.; Ershov, A. A.; Krylov, A. S.; Shaykhutdinov, K. A.; Vtyurin, A. N.; Molokeev, M. S.; Terent'ev, K. Y. and Mihashenok, N. V. Raman spectra and phase composition of $\mathrm{MnGeO}_{3}$ crystals, Journal of Raman Spectroscopy 2016, 47(5), 531-536. 
17. Gerasimova, Yu. V.; Sofronova, S. N.; Gudim, I. A.; Oreshonkov, A. S.; Vtyurin, A. N. and Ivanenko A. A. Infrared Absorption Spectra of a $\mathrm{Nd}_{0.5} \mathrm{Ho}_{0.5} \mathrm{Fe}_{3}\left(\mathrm{BO}_{3}\right)_{4}$ Crystal. Physics of Solid State 2016, 58(1), 155-159.

18. Lim, C. S.; Aleksandrovsky, A.; Molokeev, M.; Oreshonkov, A.; Atuchin, V. The modulated structure and frequency upconversion properties of $\mathrm{CaLa}_{2}\left(\mathrm{MoO}_{4}\right)_{4}: \mathrm{Ho}^{3+} / \mathrm{Yb}^{3+}$ phosphors prepared by microwave synthesis. Physical Chemistry Chemical Physics 2015, 17, 19278-19287.

19. Lim, C. S.; Aleksandrovsky, A.; Molokeev, M.; Oreshonkov, A.; Atuchin, V. Microwave sol-gel synthesis and upconversion photoluminescence properties of $\mathrm{CaGd}_{2}\left(\mathrm{WO}_{4}\right)_{4}: \mathrm{Er}^{3+} / \mathrm{Yb}^{3+}$ phosphors with incommensurately modulated structure, Journal of Solid State Chemistry 2015, 228, 160-166.

20. Savina, A. A.; Atuchin, V. V.; Solodovnikov, S. F.; Solodovnikova, Z. A.; Krylov, A. S.; Maximovskiy, E. A.; Molokeev, M. S.; Oreshonkov, A. S.; Pugachev, A. M.; Khaikina, E. G. Synthesis, structural and spectroscopic properties of acentric triple molybdate $\mathrm{Cs}_{2} \mathrm{NaBi}\left(\mathrm{MoO}_{4}\right)_{3}$, Journal of Solid State Chemistry 2015, 225, 53-58.

21. Atuchin, V. V.; Aleksandrovsky, A. S.; Chimitova, O. D.; Gavrilova, T. A.; Krylov, A. S.; Molokeev, M. S.; Oreshonkov, A. S.; Bazarov, B. G.; Bazarova, 
J. G. Synthesis and spectroscopic properties of monoclinic $\alpha-\mathrm{Eu}_{2}\left(\mathrm{MoO}_{4}\right)_{3}$, Journal of Physical Chemistry C 2014, 118, 15404-15411.

22. Xia, Zh.; Molokeev, M. S.; Oreshonkov, A. S.; Atuchin, V. V.; Liu, R.S.; Dong C. Crystal and local structure refinement in $\mathrm{Ca}_{2} \mathrm{Al}_{3} \mathrm{O}_{6} \mathrm{~F}$ explored by Xray diffraction and Raman spectroscopy. Physical Chemistry Chemical Physics 2014, 16, 5952-5957.

23. Gerasimova, Y. V.; Oreshonkov, A. S.; Vtyurin, A. N.; Ivanenko, A. A.; Isaenko, L.I.; Ershov, A. A.; Pogoreltsev, E. I. Infrared absorption investigation of the role of octahedral groups upon the phase transition in the $\mathrm{Rb}_{2} \mathrm{KMoO}_{3} \mathrm{~F}_{3}$ crystal, Physics of the Solid State 2013, 55(11), 2331-2334.

24. Krylov, A. S.; Vtyurin, A. N.; Oreshonkov, A. S.; Voronov, V. N.; Krylova, S. N. Structural transformations in single crystal $\mathrm{Rb}_{2} \mathrm{NaYF}_{6}$ : Raman scattering study, Journal of Raman Spectroscopy 2013, 44(5), 763-769.

25. Vtyurin, A. N.; Krylov, A. S.; Krylova, S. N.; Goryainov, S. V.; Voronov, V. N.; Oreshonkov, A. S. Hydrostatic pressure-induced phase transitions in $\mathrm{Rb}_{2} \mathrm{KInF}_{6}$ and $\mathrm{Rb}_{2} \mathrm{KScF}_{6}$ crystals: Raman spectra and lattice dynamics simulations. Ferroelectrics 2012, 440, 100-104. 
26. Vtyurin, A. N.; Krylov, A. S.; Goryainov, S. V.; Krylova, S. N.; Oreshonkov, A. S. and Voronov, V. N. A Raman Study of Hydrostatic Pressure Induced Phase Transitions in $\mathrm{Rb}_{2} \mathrm{KInF}_{6}$ Crystals, Physics of the Solid State 2012, 54(5), 934-936.

27. Brusentsova, T.; Peale, R. E.; Maukonen, D.; Figueiredo, P.; Harlow, G. E.; Ebel, D. S.; Nissinboim, A.; Sherman, K.; Lisse, C. M. Laboratory far-infrared spectroscopy of terrestrial sulphides to support analysis of cosmic dust spectra. Monthly Notices of the Royal Astronomical Society 2012, 420, 2569-2579.

28. Soong, R. and Farmer, V. C.; The identification of sulphide minerals by infra-red spectroscopy, Mineralogical Magazine 1978, 42, 277.

29. RRUFF database, website: http://www.rruff.info/R070174.

30. Kavc1, O.; Cabuk, S. First-principles study of structural stability, elastic and dynamical properties of MnS. Computational Materials Science 2014, 95, 99105.

31. Reich, S. and Thomsen, C. Raman spectroscopy of graphite. Philosophical Transactions of The Royal Society A 2004, 362, 2271-2288.

32. Güntherodt, G.; Grünberg, P.; Anastassakis, E.; Cardona, M.; Hackfort, H. and Zinn, W. Physical Review B 1977, 16, 3504-3512. 
33. Fernandez, J. R. L.; Souza-Parise, M.; Morais, P. C. Structural characterization and simulation of colloidal MnS. Materials Research Express 2015, 2(9), 095019.

34. Buciuman, F.; Patcas, F.; Craciun, R.; Zahn, D. R. T. Vibrational spectroscopy of bulk and supported manganese oxides. Physical Chemistry Chemical Physics 1999, 1, 185-190. 
Table 1. Experimental and calculated frequencies of modes in Raman and infrared spectra of pure $\alpha$-MnS and gadolinium-doped manganese sulfide and their assignments.

\begin{tabular}{|c|ccc|ccc|}
\hline \multirow{2}{*}{ Compound } & \multicolumn{3}{|c|}{ TO } & \multicolumn{3}{c|}{ LO } \\
\cline { 2 - 7 } & IR & R & Calc. & IR & R & Calc. \\
\hline MnS & 225 & 226 & 222 & 321 & 340 & 340 \\
$\mathrm{Gd}_{0.04} \mathrm{Mn}_{0.96} \mathrm{~S}$ & 224 & 224 & 221 & 316 & 335 & 337 \\
$\mathrm{Gd}_{0.1} \mathrm{Mn}_{0.9} \mathrm{~S}$ & 216 & - & 220 & 305 & 331 & 336 \\
$\mathrm{Gd}_{0.25} \mathrm{Mn}_{0.75} \mathrm{~S}$ & 209 & - & 216 & 289 & 327 & 322 \\
\hline
\end{tabular}




\section{Figure Captions}

Figure 1. Infrared (a.1) and Raman (b.1) spectra of pure manganese sulfide and gadolinium-doped manganese sulfide with gadolinium content equal to 0.04 (a.2, b.2), 0.1 (a.3, b.3) and 0.25 (a.4, b.4).

Fig. 2. Phonon modes of cubic gadolinium manganese sulfide solid solutions. The solid lines represent calculations of longitudinal optical and transverse optical frequencies of gadolinium doped manganese sulfide, dashed lines are longitudinal optical and transverse optical "gap” modes of gadolinium. The open squares symbols and snowflakes symbols refer to measured Raman and infrared data, respectively. Black squares are experimental Raman values of gadolinium sulfide (32).

Fig. 3. Raman spectra of gadolinium-doped manganese sulfide $(x=0.1)$ (a), Raman spectra of undamaged sample (b) Raman spectra recoded from burnt crater. 
Shortened version of the title: Spectroscopic characterization of Gd-doped MnS 